\title{
Entropy currents from holography in hydrodynamics with charge
}

\author{
Grzegorz Plewa $^{a}$ and Michat Spaliński ${ }^{a, b}$ \\ ${ }^{a}$ National Center for Nuclear Research, \\ ul. Hoża 69, 00-681 Warsaw, Poland \\ ${ }^{b}$ Physics Department, University of Biatystok, \\ ul. Lipowa 41, 15-424 Biatystok, Poland \\ E-mail: g.plewa@ipj.gov.pl, mspal@fuw.edu.pl
}

ABSTRACT: The holographic interpretation of the hydrodynamic entropy current is developed for the case of hydrodynamics with a conserved charge. This is carried out within a framework developed in earlier work [1, 2], which showed how to associate entropy currents with horizons in the dual geometry. The entropy current defined by the event horizon in the dual bulk geometry is calculated. It is also shown that to second order in the gradient expansion the dual geometry possesses a unique Weyl-invariant apparent horizon which also defines an admissable entropy current. At first order both currents coincide with the result obtained on the basis of a purely hydrodynamic analysis [3].

KEYWORDS: Gauge-gravity correspondence, Holography and quark-gluon plasmas, Black Holes in String Theory

ARXIV EPRINT: 1304.6705 


\section{Contents}

1 Introduction 1

2 The geometry of fluid-gravity duality with a conserved charge 3

3 The Weyl-invariant apparent horizon 5

4 Hydrodynamic entropy currents $\quad 9$

$\begin{array}{lll}5 & \text { Summary } & 12\end{array}$

$\begin{array}{ll}\text { A Weyl covariance } & 14\end{array}$

$\begin{array}{ll}\text { B Lorentz tensors at second order } & 14\end{array}$

$\begin{array}{ll}\text { C Second order hydrodynamics } & 15\end{array}$

$\begin{array}{ll}\text { D The expansions } & 16\end{array}$

$\begin{array}{ll}\text { E The entropy current at first order } & 18\end{array}$

\section{Introduction}

For static black holes in Einstein gravity there is a well established notion of entropy expressed in terms of the event horizon area by the Beckenstein formula [4]. One would expect that this notion should, in some form, apply also to black holes which are in an appropriate sense close to being static [5]. However, the event horizon boundary of a black hole is a global, teleological concept - its location can only be determined once the complete spacetime is known, since one must examine the ultimate fate of all signals originating from a point before ruling whether or not that point is part of the black hole [6]. In this way the evolution of an event horizon can appear to be acausal. The area of the event horizon is similarly teleological. For example the area increase of an event horizon is not directly driven by infalling matter or energy; the actual effect of an influx through the event horizon is to decrease its rate of expansion [6]. This becomes a puzzle as soon as one wishes to interpret the area of the event horizon as a measure of black hole entropy, since the apparently acausal expansion of event horizons would then seem to imply a similarly acausal evolution of entropy. An acute expression of this comes arises in the context of the AdS/CFT correspondence, which maps the entropy of an AdS black hole to the entropy of a fluid in the dual quantum field theory on the conformal boundary.

It is therefore an open question whether a naive reading of the Beckenstein formula accounts for black hole entropy for dynamical black holes. The simplest option is to assume 
that the entropy is still proportional to the area, but perhaps not to the area of the event horizon itself, but of a hypersurface which asymptotes to it at late times $[1,2,7,8]$. One such example is the apparent horizon, understood as the boundary of a region containing trapped surfaces. For static black holes the apparent and event horizons coincide, but the location of an apparent horizon on a given slicing of spacetime can be determined locally in time. Since the apparent horizon evolves in a causal way, the associated entropy also has this property. Such a notion, while not free of conceptual problems, has attracted a lot of attention [6].

In the context of the AdS/CFT correspondence the long wavelength distorsions of black hole (or black brane) horizons map to hydrodynamic states of the dual quantum field theory plasma on the AdS conformal boundary [9]. The notion of near-equilibrium entropy then maps to the hydrodynamic generalization of entropy - the entropy current $[10,11]$. This notion was introduced in the framework of relativistic hydrodynamics [12]. In the perfect fluid approximation the divergence of the entropy current vanishes on all solutions, but is non-vanishing already at first order in the gradient expansion.

Within the hydrodynamic framework the entropy current is constructed phenomenologically in the gradient expansion by requiring that in equilibrium it reproduces thermodynamic entropy and that its divergence evaluated on any solution of the equations of hydrodynamics is non-negative. A detailed analysis of the consequences of this generalized second law of thermodynamics on the form of the entropy current in [13, 14] has showed that already at second order in gradients there is an ambiguity inherent in such a definition.

It is very natural to suspect that the bulk counterpart of this ambiguity is related to the choice of horizon assumed to be the carrier. In the case of hydrodynamics with no conserved charges beyond the energy momentum tensor it was shown in $[1,2]$ that this is indeed the case. This was done by providing an explicit formula for the entropy current associated with a given hypersurface satisfying the area theorem ${ }^{1}$ and asymptoting to the event horizon at late times. This formula reproduces the result of [10] when evaluated on the event horizon.

From the perspective of the phenomenological definition of the hydrodynamic entropy current none of these hypersurfaces and none of the available bulk-boundary maps is favored over any other. However, causality of the boundary field theory seems to favor the entropy current dual to the apparent horizon - provided that it is free of the ambiguities related to foliation dependence and that the bulk-boundary map in use is causal. These issues were discussed at length in [1]. The present article applies the same methods to a more complicated, but practically important case of hydrodynamics with a conserved current. The dual holographic description of this system was established in $[16,17]$ (see also $[18,19]$ ). In this paper the solution appearing in [20] is used, which is somewhat more general in that it allows for a weakly curved boundary geometry and is presented in a gauge convenient for the task at hand.

The organization of the paper is the following. Section 2 briefly reviews the geometry dual to conformal fluid dynamics with a conserved current obtained in [20]. Section 3

\footnotetext{
${ }^{1} \mathrm{~A}$ formula of this type is also discussed in [15].
} 
describes the calculation of the relevant apparent horizon in this geometry up to second order in gradients. In section 4 the formula introduced in [1] is used to find and compare entropy currents defined by the event horizon and by the apparent horizon. A general discussion of the results and possible future directions of research is provided in section 5 . Some technical points are discussed in appendices. Appendix A provides a very brief account of Weyl invariance, and appendix B lists Weyl invariant tensors needed in the main text. Appendix $\mathrm{C}$ provides some details of the second order hydrodynamics which follow from the solution of [20]. Appendix D contains explicit results for the expansions needed for the computation of the location of the apparent horizon. Finally, appendix E relates the results obtained here to the analysis of reference [3].

\section{The geometry of fluid-gravity duality with a conserved charge}

To describe hydrodynamics with a conserved current a Maxwell field in the bulk is required $[16,17,20]$. The action of the five-dimensional Einstein-Maxwell theory under consideration reads ${ }^{2}$

$$
S=\frac{1}{16 \pi G_{N}} \int d^{5} x \sqrt{-g}\left(\frac{12}{L^{2}}+R-F^{2}-\frac{4 \kappa}{3} \epsilon^{a b c d e} A_{a} F_{b c} F_{d e}\right),
$$

where $G_{N}$ is the 5 -dimensional Newton's constant and the cosmological constant is denoted by $12 / L^{2}$. For this theory to be a consistent truncation of type IIB supergravity [21-23] the Chern-Simons coupling $\kappa$ has to assume the value $1 / 2 \sqrt{3}$.

The equations of motion derived from (2.1) support a 5-parameter family of exact, static black hole solutions $[16,17,20]$ with planar horizons obtained by boosting and dilating the AdS-Reissner-Nordstrom black brane solution [21]. The constant dilation parameter is denoted by $b$, the charge by $q$ and the boost parameter $u^{\mu}$ is a 4 -component velocity vector in the "boundary directions". ${ }^{3}$ The solution can be expressed in the form

$$
\mathrm{d} s^{2}=r^{2}\left(P_{\mu \nu}-2 B u_{\mu} u_{\nu}\right) \mathrm{d} x^{\mu} \mathrm{d} x^{\nu}-2 u_{\mu} \mathrm{d} x^{\mu} \mathrm{d} r
$$

where $^{4}$

$$
B=\frac{1}{2}\left(1-\frac{1}{b^{4} r^{4}}\left(1+q^{2} b^{6}\right)+\frac{q^{2}}{r^{6}}\right)
$$

and

$$
P_{\mu \nu}=h_{\mu \nu}+u_{\mu} u_{\nu}
$$

is the projector operator onto the space transverse to $u^{\mu}$. The lines of constant $x^{\mu}$ in $(2.2)$ are ingoing null geodesics, for large $r$ propagating in the direction set by $u^{\mu}$, and the radial coordinate $r$ parametrizes them in an affine way [10].

\footnotetext{
${ }^{2}$ Latin indices run over all spacetime dimensions, i.e. from 0 to 4 , while Greek indices run over the "boundary directions", i.e. from 0 to 3.

${ }^{3}$ The 4 -velocity vector is normalized so that $u_{\mu} u^{\mu}=-1$ in the sense of the metric on the conformal boundary of the locally asymptotically AdS spacetime (2.2), whose components are denoted by $h_{\mu \nu}$.

${ }^{4}$ The notation is chosen so that in the uncharged limit $(q \rightarrow 0) B$ is equal to $B(b r)$ as defined in [24].
} 
The vector potential takes the form:

$$
A=\frac{\sqrt{3} q}{2 r^{2}} u_{\mu} \mathrm{d} x^{\mu}
$$

The geometry (2.2) may be regarded as a stack of constant- $r$ 4-dimensional planes, starting from the conformal boundary at $r=\infty$ (which is 4-dimensional Minkowski spacetime), down to the curvature singularity at $r=0$. The latter is shielded by an event horizon at $r=1 / b$. The parameter $b$ appearing in (2.3) is related to the Hawking temperature $T$ of the event horizon by

$$
T=\frac{1}{2 \pi b}\left(2-q^{2} b^{6}\right) .
$$

It will be assumed that the charge $q$ is always below the extremal limit, so that $q^{2} b^{6}<2$.

If $b, q, u^{\mu}$ and $h_{\mu \nu}$ are allowed to vary slowly compared to the scale set by $b$, the metric (2.2) should be an approximate solution of nonlinear Einstein's equations, with corrections organized in an expansion in the number of gradients in the "boundary directions" parametrized by $x^{\mu}$. This gradient-corrected metric can be written in the form

$$
\mathrm{d} s^{2}=\left(\mathcal{G}_{\mu \nu}-2 u_{\mu} \mathcal{V}_{\nu}\right) \mathrm{d} x^{\mu} \mathrm{d} x^{\nu}-2 u_{\mu} \mathrm{d} x^{\mu}\left(\mathrm{d} r+r \mathcal{A}_{\nu} \mathrm{d} x^{\nu}\right),
$$

with the condition $u^{\mu} \mathcal{G}_{\mu \nu}=0$ completely fixing the gauge freedom [24]. The field $\mathcal{A}$ appearing in (2.7) is the "Weyl connection" defined in appendix A. It is clear that lines of constant $x$ are geodesics (affinely parametrized by $r$ ), as in the case of the static metric $(2.2)$.

The form of the metric (2.7) is strongly restricted by the Weyl-invariance ${ }^{5}$ of the bulk theory [24]: the functions $\mathcal{V}_{\mu}$ are of unit Weyl weight and $\mathcal{G}_{\mu \nu}$ are Weyl invariant. They can be expressed as linear combinations of independent Lorentz vectors and tensors built out of $b, q, u^{\mu}, h_{\mu \nu}$ and their derivatives, order by order in the gradient expansion. At first order in gradients one has a Weyl-invariant vector

$$
l_{\mu}=\epsilon_{\mu \nu \lambda \rho} u^{\nu} \mathcal{D}^{\lambda} u^{\rho},
$$

pseudovector

$$
V_{0 \mu}=q^{-1} P_{\mu}^{\nu} \mathcal{D}_{\nu} q
$$

and a second order symmetric tensor ${ }^{6}$

$$
\sigma_{\mu \nu}=\frac{1}{2} \mathcal{D}_{(\mu} u_{\nu)}
$$

of Weyl weight -1 . The independent objects appearing at second order in gradients are listed in appendix B.

As shown in [20], the resulting solution, up to second order, takes the form

$$
\begin{aligned}
\mathcal{V}_{\mu} & =r^{2} B u_{\mu}+r F_{1} l_{\mu}+b r^{2} F_{0} V_{0 \mu}+r^{2} \sum_{i=1}^{6} K_{i} S_{i} u_{\mu}+r \sum_{i=1}^{5} W_{i} V_{i \mu}, \\
\mathcal{G}_{\mu \nu} & =r^{2} P_{\mu \nu}+2 b r^{2} F_{2} \sigma_{\mu \nu}+r^{2} \sum_{i=1}^{6} L_{i} S_{i} P_{\mu \nu}+\sum_{i=1}^{11} H_{i} T_{i \mu \nu} .
\end{aligned}
$$

\footnotetext{
${ }^{5} \mathrm{~A}$ brief account of Weyl invariance appears in appendix A.

${ }^{6}$ Symmetrization is defined as $A_{(\mu \nu)}:=A_{\mu \nu}+A_{\nu \mu}$.
} 
The 31 coefficient functions $F_{0}, F_{1}, F_{2}, K_{i}, L_{i}(i=1, \ldots, 6), W_{i}(i=1, \ldots, 5)$, and $H_{i}$ $(i=1, \ldots, 11)$ all depend on the Weyl invariant variables $b^{3} q$ and $b r$.

The Maxwell gauge field $A$ is a vector field of Weyl weight zero. It will be taken in the gauge $A_{r}=0$ and its form is [20]

$$
A=\left(\frac{\sqrt{3} q u_{\mu}}{2 r^{2}}+Y_{0} l_{\mu}+\tilde{Y}_{0} V_{0 \mu}+r \sum_{i=1}^{6} N_{i} S_{i} u_{\mu}+\sum_{i=1}^{5} Y_{i} V_{i \mu}\right) \mathrm{d} x^{\mu} .
$$

As before, the coefficient functions $\tilde{Y}_{0}, Y_{0}, \ldots Y_{5}, N_{1}, \ldots, N_{6}$ depend on the Weyl invariant variables $b r$ and $b^{3} q$.

All the coefficient functions appearing in (2.11), (2.12) have been determined by solving the equations of motion ${ }^{7}$ and are given explicitly in [20].

The metric given above is a solution of the Einstein-Maxwell equations with negative cosmological constant up to second order in gradients, provided that $b, q$ and $u^{\mu}$ satisfy the equations of hydrodynamics, i.e. the equations of covariant conservation of the energymomentum tensor and charge current obtained from (2.11) and (2.12) by holographic renormalization [25]. Explicit formulae [20] can be found in appendix (C).

\section{The Weyl-invariant apparent horizon}

This section is devoted to locating the Weyl-invariant apparent horizon for the spacetimes defined by the metric (2.7) following the approach of [1, 2]. The time-evolved apparent horizon, denoted by $\Delta$, is presented as the level set of a scalar function $S(r, x)$. This function is required to be Weyl-invariant and can be written in the gradient expansion in terms of all the independent scalars available up to some order. Since the outer event horizon at order zero is at $r=1 / b$ and at that order $\Delta$ should coincide with it, one has

$$
S(r, x)=b(x) r-g(x),
$$

where $g(x)$ is a Weyl-invariant function expanded in gradients of $b, q$ :

$$
g(x)=1+g_{1}(x)+g_{2}(x)+\ldots .
$$

Here $g_{k}$ denotes a linear combination of all Weyl-invariant scalars at order $k$ in the gradient expansion. There are no Weyl-invariant scalars at order 1 , and 6 at order 2 , so one expects to find

$$
\begin{aligned}
& g_{1}(x)=0 \\
& g_{2}(x)=\sum_{k=1}^{6} h_{k}\left(b^{3} q\right) S_{k},
\end{aligned}
$$

where the $S_{i}$ are the 6 independent Weyl-invariant scalars (B.1). The functions ${ }^{8} h_{i}$ will be determined in due course by solving the condition $\theta_{\ell}=0$. Once this is done, the expression

\footnotetext{
${ }^{7}$ This, as well as most other computations in this paper, was done with the help of Mathematica.

${ }^{8}$ In principle these functions also depend on the other Weyl-invariant scalar br, but these formulae are eventually evaluated on hypersurfaces $r=1 / b$ up to terms of second order in gradients. For this reason one can set $b r=1$ in these functions, since they always appear multiplied by second order scalars.
} 
for the position of the apparent horizon will take the form

$$
r_{H}=\frac{1}{b}\left(1+\sum_{k=1}^{6} h_{k} S_{k}\right)
$$

The approach developed in $[1,2]$ starts by determining a vector field, denoted by $v$, which is normal to the outer marginally trapped surfaces which foliate the horizon $\Delta$. The two properties of $v$ which are essential to its construction are the fact that $v$ is tangent to $\Delta$, and that it must be surface forming, that is, it must satisfy the Frobenius condition

$$
v \wedge d v=0
$$

It will be shown in the following that for the geometry under consideration, up to second order in the gradient expansion, these conditions together with Weyl invariance determine $v$ uniquely.

To find the vector field $v$, one first needs the normal covector to a surface of the form (3.1). This is given by $m=d S$, which up to second order in the gradient expansion can be written in terms of the Weyl-covariant derivatives (given in (A.4), (A.5)) as

$$
m=r \mathcal{D}_{\mu} b d x^{\mu}+b\left(d r+r \mathcal{A}_{\mu} d x^{\mu}\right) .
$$

The vector $v$ is to be tangent to $\Delta$, so it must satisfy $v \cdot m=0$. To solve this condition it is convenient to make a special coordinate choice. As discussed in detail in [2], given a parametrization $y^{\mu}$ of the horizon $\Delta$ (such that $S(r(y), x(y)) \equiv$ const), one can choose the gauge $y=x$ and then the vector field $v$ takes the form

$$
v=v^{\beta}\left\{\frac{\partial}{\partial x^{\beta}}-\left(\frac{\partial S}{\partial r}\right)^{-1}\left(\frac{\partial S}{\partial x^{\beta}}\right) \frac{\partial}{\partial r}\right\} .
$$

Requiring that the vector $v$ be Weyl invariant implies that (up to second order) the "boundary" components $v^{\mu}$ take the form

$$
v^{\mu}=b\left(u^{\mu}+b\left(a_{0} V_{0}{ }^{\mu}+a_{1} l^{\mu}+\sum_{k=1}^{5} c_{k} V_{k}^{\mu}\right)+u^{\mu} \sum_{k=1}^{6} e_{k} S_{k}\right),
$$

where $a_{k}, c_{k}, e_{k}$ are some functions of the Weyl-invariant combination $b^{3} q$. Using (3.7) one finds that the $r$ component of $v$ is

$$
v^{r}=-b r A_{\mu} u^{\mu}-r u^{\mu} \mathcal{D}_{\mu} b-b r\left(b A_{\mu}+\mathcal{D}_{\mu} b\right)\left(a_{0} V_{0}{ }^{\mu}+a_{1} l^{\mu}\right) .
$$

It is computationally convenient to normalize $v$ so that

$$
m^{2}+v^{2}=0
$$

which implies in particular that coefficients of the longitudinal terms in (3.8) vanish: $e_{k}=0$ for $k=0, \ldots, 6$. 
The remaining coefficient functions appearing in $v$ are also not arbitrary. As discussed earlier, to ensure that the vector $v$ defines a foliation one has to impose the Frobenius condition (3.5). As was the case in the analysis of [2], the vanishing of $v_{[\mu} \partial_{\nu} v_{\rho]}$ is automatic, but the conditions $v_{[r} \partial_{\nu} v_{\rho]}=0$ are nontrivial. In fact these conditions determine the remaining freedom in $v$. At first order in the gradient expansion one finds

$$
\begin{aligned}
& a_{0}=-\frac{3 b^{6} q^{2}\left(b^{6} q^{2}+2\right)}{4\left(b^{12} q^{4}-b^{6} q^{2}-2\right)}, \\
& a_{1}=-\frac{\sqrt{3} b^{9} \kappa q^{3}}{b^{6} q^{2}+1}
\end{aligned}
$$

while at second order one obtains

$$
\begin{aligned}
& c_{1}=\frac{1}{b^{6} q^{2}-2}-W_{1}\left(b^{3} q, 1\right), \\
& c_{2}=\frac{1}{2}-\frac{6 b^{12} q^{4} \kappa^{2}}{\left(1+b^{6} q^{2}\right)^{2}}, \\
& c_{3}=\frac{2 \sqrt{3} b^{9} \kappa q^{3} F_{2}\left(b^{3} q, 1\right)}{b^{6} q^{2}+1}-W_{3}\left(b^{3} q, 1\right), \\
& c_{4}=\frac{3 b^{6} q^{2}}{\left(b^{6} q^{2}-2\right)^{2}}+\frac{3 b^{6} q^{2}\left(b^{6} q^{2}+2\right) F_{2}\left(b^{3} q, 1\right)}{2\left(b^{6} q^{2}-2\right)\left(b^{6} q^{2}+1\right)}-W_{4}\left(b^{3} q, 1\right), \\
& c_{5}=-W_{5}\left(b^{3} q, 1\right) .
\end{aligned}
$$

To show that the Frobenius condition (3.5) is really satisfied one also has to use the relations

$$
\begin{aligned}
\partial_{\mu} \mathcal{A}_{\nu}-\partial_{\nu} \mathcal{A}_{\mu} & =0, \\
\mathcal{D}_{\mu} V_{0 \nu}-\mathcal{D}_{\nu} V_{0 \mu} & =0
\end{aligned}
$$

(valid up to second order in gradients). These relations (discussed further in appendix C) follow from the equations of hydrodynamics. Since the Frobenius condition was imposed for the full spacetime (rather than just on the horizon), the vector $v$ actually gives rise to a foliation of the full spacetime, at least in a neighhborhood of $\Delta$.

This way one finds (as in the uncharged case [2]) that the foliation vector $v$ is completely determined once $\Delta$ is fixed. As discussed in [2], it seems plausible that this will also be the case at higher orders in the gradient expansion.

Dynamical quasilocal horizons are spacelike and so $m$ should be timelike and $v$ spacelike. Without loss of generality one can assume that $m$ is future oriented and $v$ is outward pointing. Then the null normals to the leaves of the foliation of $\Delta$ can be expressed as

$$
\begin{aligned}
v & =\ell-C n \\
m & =\ell+C n,
\end{aligned}
$$

where the scalar $C$ is called the evolution parameter $[5,26]$ :

$$
C=\frac{1}{2} v^{2}
$$


The sign of the evolution parameter indicates whether $\Delta$ is spacelike $(C>0)$, timelike $(C<0)$ or null $(C=0)$. The signs of the coefficients in (3.14) have been chosen to ensure that both $\ell$ and $n$ are future oriented, and $\ell$ is outward pointing while $n$ is inward pointing. Given the formulae for $m$ and $v$ it is straightforward to calculate $C, \ell$ and $n$ explicitly.

To determine the position of the apparent horizon one needs to calculate the null expansions

$$
\begin{aligned}
\theta_{\ell} & =\tilde{q}^{a b} \nabla_{a} \ell_{b}, \\
\theta_{n} & =\tilde{q}^{a b} \nabla_{a} n_{b},
\end{aligned}
$$

where

$$
\tilde{q}_{a b}=G_{a b}+\ell_{a} n_{b}+\ell_{b} n_{a}
$$

is the metric induced on the foliation slices and $G_{a b}$ are the components of the metric (2.7). Using the results of the previous section one finds (up to second order) ${ }^{9}$

$$
\begin{aligned}
& \theta_{\ell}=3 b B r+\sum_{k=1}^{6} \theta_{\ell}^{(k)} S_{k}, \\
& \theta_{n}=-\frac{3}{b r}+\sum_{k=1}^{6} \theta_{n}^{(k)} S_{k},
\end{aligned}
$$

where the coefficient functions $\theta_{\ell}^{(k)}$ and $\theta_{n}^{(k)}$ are listed in appendix D. Note that the results are manifestly Weyl-invariant. There is no correction at first order, as required by Weyl invariance. With these results in hand, it is straightforward to determine the location of the apparent horizon by solving $\theta_{\ell}\left(r_{A H}\right)=0$. One finds the result (3.4) with

$$
\begin{aligned}
& h_{1}^{A H}=\frac{K_{1}\left(b^{3} q, 1\right)}{b^{6} q^{2}-2}, \\
& h_{2}^{A H}=\frac{9-2 b^{6} q^{2}}{12\left(b^{6} q^{2}-2\right)}+\frac{2 b^{12} \kappa^{2} q^{4}}{5\left(b^{6} q^{2}+1\right)^{2}}, \\
& h_{3}^{A H}=\frac{1}{12\left(b^{6} q^{2}-2\right)}, \\
& h_{4}^{A H}=\frac{K_{4}\left(b^{3} q, 1\right)}{b^{6} q^{2}-2}+\frac{b^{6} q^{2}\left(b^{24} q^{8}-102 b^{18} q^{6}-244 b^{12} q^{4}-232 b^{6} q^{2}-64\right)}{32\left(b^{6} q^{2}-2\right)^{4}\left(b^{6} q^{2}+1\right)^{2}}, \\
& h_{5}^{A H}=\frac{K_{5}\left(b^{3} q, 1\right)}{b^{6} q^{2}-2}-\frac{b^{6} q^{2}\left(b^{6} q^{2}+2\right)}{4\left(b^{6} q^{2}-2\right)^{2}\left(b^{6} q^{2}+1\right)}, \\
& h_{6}^{A H}=\frac{K_{6}\left(b^{3} q, 1\right)}{b^{6} q^{2}-2}+\frac{\sqrt{3} b^{9} \kappa q^{3}\left(3 b^{12} q^{4}+14 b^{6} q^{2}+8\right)}{4\left(b^{6} q^{2}-2\right)^{2}\left(b^{6} q^{2}+1\right)^{2}} .
\end{aligned}
$$

In the uncharged case considered in $[1,2]$, only $h_{1}$ differs from the result for the event horizon. In the present case, the event horizon determined in [20] differs from the apparent

\footnotetext{
${ }^{9}$ This computation is fairly lengthy.
} 
horizon in the coefficients $h_{1}$ and $h_{4}$ :

$$
\begin{aligned}
& h_{1}^{(E H)}=h_{1}^{(A H)}+\frac{1}{3\left(b^{6} q^{2}-2\right)^{2}} \\
& h_{4}^{(E H)}=h_{4}^{(A H)}+\frac{b^{6} q^{2}\left(b^{6} q^{2}+2\right)^{2}}{2\left(b^{6} q^{2}-2\right)^{4}} .
\end{aligned}
$$

The expression

$$
r_{E H}-r_{A H}=\frac{1}{b} \frac{1}{\left(b^{6} q^{2}-2\right)^{2}}\left(\frac{1}{3} S_{1}+\frac{b^{6} q^{2}}{2} \frac{\left(b^{6} q^{2}+2\right)^{2}}{\left(b^{6} q^{2}-2\right)^{2}} S_{4}\right) \geq 0
$$

explicitly shows ${ }^{10}$ that the apparent horizon lies within (or coincides with) the event horizon in the sense that an ingoing radial null geodesic will cross first the event horizon and only then the apparent horizon, since $r$ is an affine parameter on such geodesics. It is also easy to check that (in accordance with expectations) the apparent horizon is spacelike or null

$$
C\left(r_{A H}\right)=\frac{1}{\left(2-b^{6} q^{2}\right)}\left(\frac{1}{3} S_{1}+\frac{b^{6} q^{2}}{2} \frac{\left(b^{6} q^{2}+2\right)^{2}}{\left(b^{6} q^{2}-2\right)^{2}} S_{4}\right) \geq 0
$$

as long as the black brane is subextremal (that is, $b^{6} q^{2}<2$ ).

\section{Hydrodynamic entropy currents}

In hydrodynamics the entropy current is a phenomenological notion constructed order-byorder in the gradient expansion. The leading term describes the flow of thermodynamic entropy in the perfect fluid approximation. In the case considered in this paper, the most general form of the entropy current consistent with conformal symmetry up to second order in gradients reads

$$
S^{\mu}=\frac{1}{4 G_{N}} b^{-3}\left(u^{\mu}+b\left(j_{0} V_{0}^{\mu}+j_{1} l^{\mu}+\sum_{k=1}^{5} j_{k}^{\perp} V_{k}^{\mu}\right)+\left(\sum_{k=1}^{6} j_{k}^{\|} S_{k}\right) u^{\mu}\right) .
$$

The overall factor of $1 / 4 G_{N}$ in (4.1) comes from the holographic formula for thermodynamic entropy. The coefficients $j_{0}, j_{1}, j_{k}^{\perp}$ and $j_{k}^{\|}$appearing in this expression are functions of the Weyl-invariant variable $b^{3} q$ and should be such as to ensure that the divergence of this current is non-negative on all solutions of the equations of hydrodynamics. For the case without charge this condition was analyzed by [27], where it was found that it is not possible to determine all these coefficients in terms of the transport coefficients without some additional input. Thus, if the notion of local entropy production in the nearequilibrium regime makes sense, there must be some further constraints on the form of the hydrodynamic entropy current.

This prompted the analysis of [1], which showed that the ambiguity in the entropy current is reflected on the gravity side precisely as an ambiguity in the choice of horizon

\footnotetext{
${ }^{10}$ Both $S_{1}$ and $S_{4}$ are manifestly positive.
} 
used to define the entropy current. In [1] a formula was proposed, which associates an entropy current with each hypersurface which satisfies the Hawking area increase theorem, such as the event or apparent horizons:

$$
S^{\mu}=\frac{1}{4 G_{N}} \frac{1}{b} \sqrt{\frac{G}{h}} v^{\mu},
$$

where $G$ is the determinant of the bulk metric (2.7) and $h$ is the determinant of the weakly curved boundary metric. This formula, as discussed at length in [1], is to be evaluated on the chosen horizon. The divergence of this current was shown to be proportional to the quantity $\theta_{\ell}-C \theta_{n}$, which is non-negative on the basis of the area theorem.

To evaluate the entropy current according to (4.2) one needs to calculate the determinant of the metric (2.7). This determinant is proportional to $h$; up to terms of higher order in the gradient expansion one finds

$$
\frac{G}{h}=r^{6}\left(1+\left(3 L_{1}-2 F_{2}^{2}\right) S_{1}+\left(\frac{1}{b^{2} r^{2}}-\frac{12 b^{6} \kappa^{2} q^{4}}{5 r^{6}\left(b^{6} q^{2}+1\right)^{2}}\right) S_{2}+3\left(L_{4} S_{4}+L_{5} S_{5}+L_{6} S_{6}\right)\right) .
$$

Using this, as well as the vector $v$ determined earlier, one can evaluate (4.2) on a hypersurface of the form (3.4). This leads to the result (4.1). Coefficients of the first order terms are found to be

$$
\begin{aligned}
& j_{0}=-\frac{3 b^{6} q^{2}\left(b^{6} q^{2}+2\right)}{4\left(b^{6} q^{2}-2\right)\left(b^{6} q^{2}+1\right)}, \\
& j_{1}=-\frac{\sqrt{3} b^{9} \kappa q^{3}}{b^{6} q^{2}+1} .
\end{aligned}
$$

Coefficients of the transverse second order terms are given by

$$
\begin{aligned}
j_{1}^{\perp} & =\frac{1}{b^{6} q^{2}-2}-W_{1}\left(b^{3} q, 1\right) \\
j_{2}^{\perp} & =\frac{1}{2}-\frac{6 b^{12} \kappa^{2} q^{4}}{\left(b^{6} q^{2}+1\right)^{2}} \\
j_{3}^{\perp} & =\frac{2 \sqrt{3} b^{9} \kappa q^{3} F_{2}\left(b^{3} q, 1\right)}{b^{6} q^{2}+1}-W_{3}\left(b^{3} q, 1\right) \\
j_{4}^{\perp} & =\frac{3 b^{4} q^{2}}{\left(b^{6} q^{2}-2\right)^{2}}+\frac{3 b^{4} q^{2}\left(b^{6} q^{2}+2\right) F_{2}\left(b^{3} q, 1\right)}{2\left(b^{6} q^{2}-2\right)\left(b^{6} q^{2}+1\right)}-W_{4}\left(b^{3} q, 1\right) \\
j_{5}^{\perp} & =-W_{5}\left(b^{3} q, 1\right)
\end{aligned}
$$

These are all fixed independently of the hypersurface on which the formula (4.2) is evaluated. The coefficient functions of the longitudinal terms, proportional to the 4 -velocity $u^{\mu}$, 
do however depend on the choice of horizon through the functions $h_{k}$ :

$$
\begin{aligned}
j_{1}^{\|} & =-F_{2}\left(b^{3} q, 1\right)^{2}+\frac{3}{2} L_{1}\left(b^{3} q, 1\right)+3 h_{1}, \\
j_{2}^{\|} & =\frac{1}{2}-\frac{6 b^{12} q^{4} \kappa^{2}}{5\left(1+b^{6} q^{2}\right)^{2}}+3 h_{2}, \\
j_{3}^{\|} & =3 h_{3}, \\
j_{4}^{\|} & =\frac{3}{2} L_{4}\left(b^{3} q, 1\right)+3 h_{4}, \\
j_{5}^{\|} & =\frac{3}{2} L_{5}\left(b^{3} q, 1\right)+3 h_{5}, \\
j_{6}^{\|} & =\frac{3}{2} L_{6}\left(b^{3} q, 1\right)+3 h_{6} .
\end{aligned}
$$

Evaluating (4.2) on the event horizon found in [20] leads to the result

$$
\begin{aligned}
& j_{1}^{\|}=-F_{2}\left(b^{3} q, 1\right)^{2}+\frac{3}{2} L_{1}\left(b^{3} q, 1\right)+\frac{3 K_{1}\left(b^{3} q, 1\right)}{b^{6} q^{2}-2}+\frac{1}{\left(b^{6} q^{2}-2\right)^{2}}, \\
& j_{2}^{\|}=\frac{5}{4 b^{6} q^{2}-8}, \\
& j_{3}^{\|}=\frac{1}{4 b^{6} q^{2}-8}, \\
& j_{4}^{\|}=\frac{3}{2} L_{4}\left(b^{3} q, 1\right)+\frac{3 K_{4}\left(b^{3} q, 1\right)}{b^{6} q^{2}-2}+\frac{3 b^{12} q^{4}\left(17 b^{12} q^{4}+28 b^{6} q^{2}+20\right)}{32\left(b^{6} q^{2}-2\right)^{3}\left(b^{6} q^{2}+1\right)^{2}}, \\
& j_{5}^{\|}=-\frac{3 b^{6} q^{2}\left(b^{6} q^{2}+2\right)}{4\left(b^{6} q^{2}-2\right)^{2}\left(b^{6} q^{2}+1\right)}+\frac{3}{2} L_{5}\left(b^{3} q, 1\right)+\frac{3 K_{5}\left(b^{3} q, 1\right)}{b^{6} q^{2}-2}, \\
& j_{6}^{\|}=\frac{3 \sqrt{3} b^{9} \kappa q^{3}\left(3 b^{12} q^{4}+14 b^{6} q^{2}+8\right)}{4\left(b^{6} q^{2}-2\right)^{2}\left(b^{6} q^{2}+1\right)^{2}}+\frac{3}{2} L_{6}\left(b^{3} q, 1\right)+\frac{3 K_{6}\left(b^{3} q, 1\right)}{b^{6} q^{2}-2} .
\end{aligned}
$$

For the apparent horizon one finds the same results as above apart from the coefficient functions $j_{1}^{\|}$and $j_{4}^{\|}$:

$$
\begin{aligned}
& j_{1}^{\|(E H)}-j_{1}^{\|(A H)}=\frac{1}{\left(b^{6} q^{2}-2\right)^{2}}, \\
& j_{4}^{\|(E H)}-j_{4}^{\|(A H)}=3 \frac{b^{6} q^{2}}{2} \frac{\left(b^{6} q^{2}+2\right)^{2}}{\left(b^{6} q^{2}-2\right)^{4}} .
\end{aligned}
$$

In the limit of vanishing charge one can check that the results of $[1,2]$ are reproduced. ${ }^{11}$

The fact that the two entropy currents considered here differ in the coefficients of both $S_{1}$ and $S_{4}$ suggests that in this case there is a two parameter family of entropy currents satisfying all the hydrodynamic criteria. In the case without charge there was only a one

\footnotetext{
${ }^{11}$ Note however that in the published version of [2] there is a sign error in eq. (81).
} 
parameter family ${ }^{12}$ — this was shown by hydrodynamic arguments by Romatschke [27], but the generalization of this reasoning to hydrodynamics with charge is not known at present.

The second order contribution to the entropy current cannot be expressed uniquely in terms of the transport coefficients. At first order however there is no ambiguity, and indeed the result obtained above is unique at that order. One can easily see that this result is consistent with the purely hydrodynamic analysis of Son and Surówka [3] (see also [28]), who expressed the first order entropy current in terms of the transport coefficients. This is discussed further in appendix E.

According to the analysis of [1], the divergence of the entropy current is proportional to the quantity $\theta_{\ell}-C \theta_{n}$ evaluated on the relevant horizon. Calculating this expression on either one of the horizons up to second order in gradients, one obtains

$$
\theta_{\ell}-\left.C \theta_{n}\right|_{r=r_{A H}}=\frac{3}{\left(2-b^{6} q^{2}\right)}\left(\frac{1}{3} S_{1}+\frac{b^{6} q^{2}}{2} \frac{\left(b^{6} q^{2}+2\right)^{2}}{\left(b^{6} q^{2}-2\right)^{2}} S_{4}\right),
$$

which matches the hydrodynamic result. Thus, as expected on the basis of the area theorem, the divergence of both entropy currents considered here is non-negative, assuming that the charge remains sub-extremal (i.e. $b^{6} q^{2}<2$ ).

It is interesting to observe that the second order divergence of the entropy current computed above is proportional to the separation of the event and apparent horizons (3.23). This is not an accident, since the event horizon coincides with the apparent horizon in equilibrium, so the divergence of the entropy current has to vanish when the separation of the two horizons goes to zero. The second order divergence of the entropy current must therefore be proportional to the separation computed to second order. At higher orders it must still be true that the divergence is proportional to the separation, but it is not clear whether the coefficient would be a zeroth order quantity.

The divergence of the full second order entropy current is of third order in gradients, so calculating it by evaluating the quantity $\theta_{\ell}-C \theta_{n}$ (by a bulk computation) would require determining the geometry to third order in gradients, which has so far not been done.

\section{Summary}

The ambiguity in the definition of the entropy current in relativistic second order hydrodynamics was recently connected with the issue of dynamical black hole boundaries [1]. This was based on the holographic representation of strongly coupled supersymmetric YangMills plasma in the framework of fluid-gravity duality [9, 10]. Reference [1] proposed an explicit formula which associates an entropy current with a horizon in the bulk geometry. As long as the horizon satisfies the area theorem, the divergence of the corresponding hydrodynamic entropy current is non-negative.

In references $[1,2]$ entropy currents defined by the event horizon and the Weyl-invariant apparent horizon were considered in the case of conformal hydrodynamics with no conserved

\footnotetext{
${ }^{12}$ Only parameters which appear in the divergence of the entropy current appear to be significant, and only such are considered in this counting.
} 
charges beyond the energy-momentum tensor. This line of research was continued here by studying the holographic representation of entropy currents in the case of hydrodynamics with a conserved charge $[16,17,20]$. As in $[1,2]$, currents associated with two horizons were considered: the event horizon (whose location in the relevant geometry was established in [20]) and the Weyl-invariant apparent horizon, which was found in this paper using the novel approach to locating apparent horizons developed in [2] . This method is particularly suited to situations, where an apparent horizon which respects a given symmetry is sought. The work reported here provides another (much more complex) example where this approach can successfully be applied.

The key idea behind [2] is that the apparent horizons of interest in the context of fluidgravity duality are only those which are covariant in the sense of the dual hydrodynamic description. In the case considered here this means they are specified covariantly (in the boundary sense) in terms of $b, q, u^{\mu}$ and their gradients. An important further constraint in the present context is Weyl invariance.

One of the main results of this paper is that up to second order in gradients a unique Weyl-invariant apparent horizon exists which is covariant in this hydrodynamic sense. ${ }^{13}$ As in the case without a conserved charge, this horizon is isolated at leading and first subleading orders of the gradient expansion and becomes spatial once second order gradient contributions are included. As in [1], this apparent horizon gives rise to a notion of hydrodynamic entropy current which satisfies all the hydrodynamic constraints. ${ }^{14}$

At first order in gradients the entropy current in hydrodynamics with charge was considered by Son and Surówka [3]. They carefully analyzed hydrodynamic constraints, allowing for the possibility of a $U(1)$ anomaly, and succeeded in expressing the entropy current at first order in terms of the transport coefficients. As shown in section 4, the result of this purely hydrodynamic analysis is reproduced by the holographic entropy current formula proposed in reference [1].

At second order, in the case of conformal hydrodynamics without charge, a purely hydrodynamic analysis of the allowed form of entropy currents was performed in [27] (see also [14]). The current associated with the apparent horizon was found to be consistent with that analysis [1]. In the case with charge such an analysis has not yet been carried out; it would furnish an nontrivial check on the results obtained in this paper, which imply that in hydrodynamics with a charge current there should be at least a two parameter family of entropy currents at second order in gradients.

It would also be very interesting to study apparent horizons in the cases of nonconformal [29], and superfluid [30, 31] fluid-gravity dualities and explore the issue of entropy currents in those contexts. Another possible direction of further research would be to include background fields along the lines of [18, 32, 33].

\footnotetext{
${ }^{13}$ The arguments given in [2] suggesting that the uniqueness of the Weyl-invariant apparent horizon persists at higher orders apply also here.

${ }^{14}$ The issue of the bulk-to-boundary map, introduced in [10] was further discussed in [1, 2]. The present work brings nothing new in this regard.
} 


\section{Acknowledgments}

The authors would like to thank Michał P. Heller for discussions and helpful comments on the manuscript, and Kasper Peeters for his excellent package Cadabra [34, 35]. This work was partially supported by Polish Ministry of Science and Higher Education grant N N202 173539.

\section{A Weyl covariance}

Conformal symmetry of $\mathcal{N}=4$ supersymmetric Yang-Mills theory can be extended to the bulk as follows $[24,36]$ :

$$
g_{\mu \nu} \rightarrow e^{-2 \phi} g_{\mu \nu}, \quad u^{\mu} \rightarrow e^{\phi} u^{\mu}, \quad b \rightarrow e^{-\phi} b \quad \text { and } \quad r \rightarrow e^{\phi} r
$$

where $\phi$ depends on the coordinates $x^{\mu}$ [24]. A quantity which transforms homogeneously with a factor of $e^{w \phi}$ is said to transform with Weyl weight $w$.

A beautiful formalism allowing for manifest Weyl covariance in conformal hydrodynamics was introduced by Loganayagam [11] and applied to fluid-gravity duality in [24, 36]. The basic tool is the Weyl-covariant derivative $\mathcal{D}_{\mu}$, which preserves the Weyl weight of the differentiated tensor. It is constructed using the vector field $\mathcal{A}_{\nu}$ defined by [11]

$$
\mathcal{A}_{\nu} \equiv u^{\lambda} \nabla_{\lambda} u_{\nu}-\frac{\nabla_{\lambda} u^{\lambda}}{3} u_{\nu}
$$

This quantity is of order one in the gradient expansion and transforms as a connection under Weyl-transformations

$$
\mathcal{A}_{\nu} \rightarrow \mathcal{A}_{\nu}+\partial_{\nu} \phi
$$

Due to this property it can be used to compensate for derivatives of the Weyl factor when differentiating a Weyl-covariant tensor. For instance, one has

$$
\begin{aligned}
& \mathcal{D}_{\mu} b=\partial_{\mu} b-\mathcal{A}_{\mu} b, \\
& \mathcal{D}_{\mu} q=\partial_{\mu} q+3 \mathcal{A}_{\mu} q .
\end{aligned}
$$

For further details the reader is referred to the original literature cited above.

\section{B Lorentz tensors at second order}

- Scalars:

$$
\begin{aligned}
& S_{1}=b^{2} \sigma_{\mu \nu} \sigma^{\mu \nu} \\
& S_{2}=b^{2} \omega_{\mu \nu} \omega^{\mu \nu} \\
& S_{3}=b^{2} \mathcal{R} \\
& S_{4}=b^{2} q^{-2} P^{\mu \nu} \mathcal{D}_{\mu} q \mathcal{D}_{\nu} q, \\
& S_{5}=b^{2} q^{-1} P^{\mu \nu} \mathcal{D}_{\mu} \mathcal{D}_{\nu} q, \\
& S_{6}=b^{2} q^{-1} P^{\mu \nu} l_{\mu} \mathcal{D}_{\nu} q .
\end{aligned}
$$


- Vectors:

$$
\begin{aligned}
V_{1 \mu} & =b P_{\mu \nu} \mathcal{D}_{\rho} \sigma^{\nu \rho}, \\
V_{2 \mu} & =b P_{\mu \nu} \mathcal{D}_{\rho} \omega^{\nu \rho}, \\
V_{3 \mu} & =b l^{\lambda} \sigma_{\mu \lambda}, \\
V_{4 \mu} & =b q^{-1} \sigma_{\mu}{ }^{\alpha} \mathcal{D}_{\alpha} q, \\
V_{5 \mu} & =b q^{-1} \omega_{\mu}{ }^{\alpha} \mathcal{D}_{\alpha} q .
\end{aligned}
$$

- Tensors:

$$
\begin{aligned}
T_{1 \mu \nu} & =u^{\rho} \mathcal{D}_{\rho} \sigma_{\mu \nu}, \\
T_{2 \mu \nu} & =C_{\mu \alpha \nu \beta} u^{\alpha} u^{\beta}, \\
T_{3 \mu \nu} & =\omega_{\mu}^{\lambda} \sigma_{\lambda \nu}+\omega_{\nu}{ }^{\lambda} \sigma_{\lambda \mu}, \\
T_{4 \mu \nu} & =\sigma_{\mu}{ }^{\lambda} \sigma_{\lambda \nu}-\frac{1}{3} P_{\mu \nu} \sigma_{\alpha \beta} \sigma^{\mu \nu}, \\
T_{5 \mu \nu} & =\omega_{\mu}^{\lambda} \omega_{\lambda \nu}+\frac{1}{3} P_{\mu \nu} \omega_{\alpha \beta} \omega^{\alpha \beta}, \\
T_{6 \mu \nu} & =\Pi_{\mu \nu}^{\alpha \beta} \mathcal{D}_{\alpha} l_{\beta}, \\
T_{7 \mu \nu} & =\frac{1}{2} \epsilon^{\alpha \beta}{ }_{\lambda(\mu} C_{\alpha \beta \nu) \sigma} u^{\lambda} u^{\sigma}, \\
T_{8 \mu \nu} & =q^{-2} \Pi_{\mu \nu}^{\alpha \beta} \mathcal{D}_{\alpha} q \mathcal{D}_{\beta} q, \\
T_{9 \mu \nu} & =q^{-1} \Pi_{\mu \nu}^{\alpha \beta} \mathcal{D}_{\alpha} \mathcal{D}_{\beta} q, \\
T_{10 \mu \nu} & =q^{-1} \Pi_{\mu \nu}^{\alpha \beta} l_{\alpha} \mathcal{D}_{\beta} q, \\
T_{11 \mu \nu} & =\frac{1}{2} \epsilon_{(\mu}^{\alpha \beta \lambda} \sigma_{\nu) \lambda} u_{\alpha} q^{-1} \mathcal{D}_{\beta} q .
\end{aligned}
$$

Here $\Pi_{\mu \nu}^{\alpha \beta}$ is the projector which can be used to create symmetric, traceless tensors:

$$
\Pi_{\mu \nu}^{\alpha \beta}=\frac{1}{2}\left(P_{\mu}^{\alpha} P_{\nu}^{\beta}+P_{\nu}^{\alpha} P_{\mu}^{\beta}-\frac{2}{3} P^{\alpha \beta} P_{\mu \nu}\right) .
$$

The scalar $\mathcal{R}$ is defined as in [24] and $C_{\mu \alpha \nu \beta}$ denotes the Weyl tensor:

$$
C_{\mu \nu \lambda \sigma}=R_{\mu \nu \lambda \sigma}-\frac{1}{d-2}\left(h_{\mu[\lambda} R_{\sigma] \nu}-h_{\nu[\lambda} R_{\sigma] \mu}\right)+\frac{1}{(d-1)(d-2)} h_{\mu[\lambda} h_{\sigma] \nu} R .
$$

\section{Second order hydrodynamics}

As shown in [20] the validity of the gradient expanded solution requires the conservation of

$$
\begin{aligned}
T_{\mu \nu} & =\frac{1}{16 \pi G_{N}}\left(\frac{1+b^{6} q^{2}}{b^{4}}\left(P_{\mu \nu}+3 u_{\mu} u_{\nu}\right)-\frac{2 \sigma_{\mu \nu}}{b^{3}}+\frac{2\left(1+c_{1}\right) T_{1 \mu \nu}}{b^{2}}+\frac{2 T_{2 \mu \nu}}{b^{2}}+\frac{2 c_{1} T_{3 \mu \nu}}{b^{2}}+\right. \\
& +\frac{2 T_{4 \mu \nu}}{b^{2}}+\frac{4 b^{4} q^{2}\left(-1+b^{6} q^{2}\left(12 \kappa^{2}-1\right)\right)}{1+b^{6} q^{2}} T_{5 \mu \nu}+\frac{2 \sqrt{3} b^{7} q^{3} \kappa}{1+b^{6} q^{2}} T_{6 \mu \nu}+\frac{c_{8}}{b^{2}} T_{8 \mu \nu}+ \\
& \left.+\frac{c_{9}}{b^{2}} T_{9 \mu \nu}+\frac{c_{10}}{b^{2}} T_{10 \mu \nu}\right)
\end{aligned}
$$


and

$$
\begin{aligned}
J_{\mu}= & \frac{1}{8 \pi G_{N}}\left(\frac{\sqrt{3} q u_{\mu}}{2}+\frac{3 b^{4} q^{2} \kappa l_{\mu}}{2\left(1+b^{6} q^{2}\right)}-\frac{\sqrt{3} b^{3} q\left(2+b^{6} q^{2}\right)}{8\left(1+b^{6} q^{2}\right) b^{2}} V_{0 \mu}+\frac{3 \sqrt{3} b q}{8\left(1+b^{6} q^{2}\right)} V_{1 \mu}+\right. \\
& +\frac{3 \sqrt{3} b^{7} q^{3} \kappa^{2}}{\left(1+b^{6} q^{2}\right)^{2}} V_{2 \mu}-\frac{3 b^{4} \kappa q^{2}}{2\left(b^{6} q^{2}+1\right)^{2}} V_{3 \mu}+\frac{2 a_{4}\left(b^{6} q^{2}+1\right)+\sqrt{3} b^{9} q^{3}}{16 b^{2}\left(b^{6} q^{2}+1\right)^{2}} V_{4 \mu}+ \\
& \left.+\frac{a_{5}\left(b^{6} q^{2}+1\right)+\sqrt{3} b^{9}\left(24 \kappa^{2}-1\right) q^{3}-\sqrt{3} b^{3} q}{8 b^{2}\left(b^{6} q^{2}+1\right)^{2}} V_{5 \mu}\right)
\end{aligned}
$$

where all the quantities appearing above are given in [20].

This leads to the equations of hydrodynamics

$$
\begin{aligned}
\mathcal{D}_{\mu} b= & \frac{b^{7} q^{2} V_{0 \mu}}{2-b^{6} q^{2}}+\frac{b V_{1 \mu}}{b^{6} q^{2}-2}+\frac{3 b^{7} q^{2} V_{4 \mu}}{\left(b^{6} q^{2}-2\right)^{2}}+\frac{S_{1} u_{\mu}}{6-3 b^{6} q^{2}}-\frac{b^{12} q^{4} S_{4} u_{\mu}}{4\left(b^{6} q^{2}-2\right)\left(b^{6} q^{2}+1\right)}+ \\
& +\frac{b^{6} q^{2}\left(b^{6} q^{2}+2\right) S_{5} u_{\mu}}{4\left(b^{6} q^{2}-2\right)\left(b^{6} q^{2}+1\right)}-\frac{2 \sqrt{3} b^{9} \kappa q^{3} S_{6} u_{\mu}}{\left(b^{6} q^{2}-2\right)\left(b^{6} q^{2}+1\right)} \\
\mathcal{D}_{\mu} q= & q V_{0 \mu}+\frac{b^{5} q^{3} S_{4} u_{\mu}}{4 b^{6} q^{2}+4}-\frac{q\left(b^{6} q^{2}+2\right) S_{5} u_{\mu}}{4\left(b^{7} q^{2}+b\right)}+\frac{2 \sqrt{3} b^{2} \kappa q^{2} S_{6} u_{\mu}}{b^{6} q^{2}+1}
\end{aligned}
$$

From these it follows that

$$
\begin{aligned}
\partial_{\mu} \mathcal{A}_{\nu}-\partial_{\nu} \mathcal{A}_{\mu} & =\frac{b^{6} q^{2}}{2-b^{6} q^{2}}\left(\mathcal{D}_{\nu} V_{0 \mu}-\mathcal{D}_{\mu} V_{0 \nu}\right), \\
\partial_{\mu} \mathcal{A}_{\nu}-\partial_{\nu} \mathcal{A}_{\mu} & =-\frac{1}{3}\left(\mathcal{D}_{\nu} V_{0 \mu}-\mathcal{D}_{\mu} V_{0 \nu}\right),
\end{aligned}
$$

which leads to the relations (3.13) used earlier.

\section{The expansions}

The coefficients appearing in the expansion $\theta_{\ell}$ are: ${ }^{15}$

$$
\begin{aligned}
\theta_{l}^{1}= & 3 b r K_{1}-2 b^{2} B r^{2} F_{2} F_{2}{ }^{\prime}+\frac{3}{2} b^{2} B r^{2} L_{1}{ }^{\prime}, \\
\theta_{l}^{2}= & \frac{5 b^{6} q^{2} r^{2}+b^{4}\left(4 r^{6}-7 q^{2}\right)+5 r^{2}}{4 b^{5} r^{7}}+\frac{3 b^{3} \kappa^{2} q^{4}}{5 r^{9}\left(b^{6} q^{2}+1\right)^{2}\left(b^{4} q^{2}-b^{2} r^{4}-r^{2}\right)}( \\
& +10 b^{20} q^{4} r^{10}-5 b^{18} q^{4} r^{8}-5 b^{16} q^{4} r^{6}+5 b^{14} q^{2} r^{4}\left(q^{2}+2 r^{6}\right)+5 b^{12} q^{2} r^{2}\left(q^{2}-4 r^{6}\right)+ \\
& \left.-4 b^{10}\left(2 q^{4}+5 q^{2} r^{6}\right)+12 b^{8} q^{2} r^{4}+9 b^{6}\left(2 q^{2} r^{2}-r^{8}\right)-b^{4}\left(8 q^{2}+9 r^{6}\right)+13 b^{2} r^{4}+13 r^{2}\right), \\
\theta_{l}^{3}= & \frac{1}{4 b r},
\end{aligned}
$$

\footnotetext{
${ }^{15}$ The prime denotes a derivative with respect to $b r$.
} 


$$
\begin{aligned}
& \theta_{l}^{4}=\frac{b^{7} q^{2}}{32 r\left(b^{6} q^{2}-2\right)^{3}\left(b^{6} q^{2}+1\right)^{2}\left(1-b^{2} r^{2}\right)\left(-b^{6} q^{2}+b^{2} r^{2}+b^{4} r^{4}\right)}( \\
& 18 b^{8} q^{2} r^{4}\left(b^{6} q^{2}-2\right)\left(b^{6} q^{2}+1\right)\left(b^{6} q^{2}+2\right)^{2}-12 b r^{3}\left(b^{6} q^{2}+1\right)^{2}\left(b^{18} q^{6}+14 b^{12} q^{4}+12 b^{6} q^{2}+8\right)+ \\
& -32 b^{4} r^{6}\left(b^{6} q^{2}+1\right)^{2}\left(b^{12} q^{4}-7 b^{6} q^{2}-2\right)+16 b^{4}\left(b^{6} q^{3}+q\right)^{2}\left(b^{12} q^{4}+8 b^{6} q^{2}+4\right)+ \\
& -r^{2}\left(b^{6} q^{2}\left(9 b^{24} q^{8}+214 b^{18} q^{6}+580 b^{12} q^{4}+456 b^{6} q^{2}+64\right)+64\right)+ \\
& +12 b^{5} r^{7}\left(b^{6} q^{2}+1\right)\left(b^{18} q^{6}+14 b^{12} q^{4}+12 b^{6} q^{2}+8\right)+ \\
& \left.-12 b^{5} q^{2} r\left(b^{6} q^{2}+1\right)\left(b^{18} q^{6}-18 b^{12} q^{4}-20 b^{6} q^{2}+8\right)\right)+ \\
& +\frac{b^{2} F_{0}}{4\left(b^{6} q^{2}-2\right)\left(b^{6} q^{2}+1\right)\left(1-b^{2} r^{2}\right)\left(-b^{6} q^{2}+b^{2} r^{2}+b^{4} r^{4}\right)}\left(2 b^{18} q^{6} r^{2}-3 b^{17} q^{6} r+\right. \\
& \left.+2 b^{16}\left(q^{6}-5 q^{4} r^{6}\right)+6 b^{10}\left(q^{4}-q^{2} r^{6}\right)-6 b^{6} q^{2} r^{2}+12 b^{5} q^{2} r+4 b^{4}\left(q^{2}+r^{6}\right)-4 r^{2}\right)+ \\
& +3 b r K_{4}+\frac{b^{3} q\left(b^{6} q^{2}+1\right)}{b^{6} q^{2}-2} \frac{\partial F_{0}}{\partial\left(b^{3} q\right)}+\frac{3}{2} b^{2} B r^{2} L_{4}{ }^{\prime}+\frac{b^{3} r^{3}\left(b^{6} q^{2}-3 b^{4} r^{4}+1\right) F_{0}{ }^{2}}{2\left(1-b^{2} r^{2}\right)\left(-b^{6} q^{2}+b^{2} r^{2}+b^{4} r^{4}\right)}, \\
& \theta_{l}^{5}=-\frac{1}{2} F_{0}+3 b r K_{5}+\frac{3}{2} b^{2} B r^{2} L_{5}{ }^{\prime}-\frac{b^{5} q^{2}\left(3 b^{7} q^{2} r+4 b^{6} q^{2}+6 b r+4\right)}{8 r\left(b^{6} q^{2}-2\right)\left(b^{6} q^{2}+1\right)}, \\
& \theta_{l}^{6}=\frac{3 \sqrt{3} b^{3} \kappa q^{3}}{4 r^{6}\left(b^{6} q^{2}-2\right)\left(b^{6} q^{2}+1\right)^{2}\left(b^{4} q^{2}-b^{2} r^{4}-r^{2}\right)}\left(2 b^{23} q^{6} r^{7}+b^{17} q^{4} r\left(q^{2}+6 r^{6}\right)+4 b^{16} q^{4} r^{6}-3 b^{15} q^{4} r^{5}\right. \\
& -4 b^{14} q^{2} r^{10}-3 b^{13} q^{4} r^{3}-4 b^{12} q^{2} r^{8}+4 b^{11} q^{2} r^{7}+4 b^{10} q^{2}\left(q^{2}+r^{6}\right)-6 b^{9} q^{2} r^{5}-4 b^{8} r^{4}\left(q^{2}+r^{6}\right)+ \\
& \left.-6 b^{7} q^{2} r^{3}-4 b^{6} r^{2}\left(q^{2}+r^{6}\right)-4 b^{5} q^{2} r+4 b^{4} q^{2}-4 b^{2} r^{4}-4 r^{2}\right)+3 b r K_{6}+\frac{3}{2} b^{2} B r^{2} L_{6}{ }^{\prime} .
\end{aligned}
$$

For $\theta_{n}$ the result is

$$
\begin{aligned}
\theta_{n}^{1}= & 2 F_{2} F_{2}{ }^{\prime}-\frac{3}{2} L_{1}{ }^{\prime}, \\
\theta_{n}^{2}= & \frac{1}{b^{3} r^{3}}+\frac{6 b^{2} \kappa^{2} q^{4}}{5 r^{7}\left(b^{6} q^{2}+1\right)^{2}\left(-b^{4} q^{2}+b^{2} r^{4}+r^{2}\right)^{2}}( \\
& b^{3}\left(9 b^{8} q^{4}+10 b^{12} q^{2} r^{10}\left(b^{6} q^{2}+1\right)+r^{4}\left(5 b^{12} q^{4}-28 b^{6} q^{2}-6\right)+\right. \\
& \left.\left.+b^{4} r^{8}\left(5 b^{6} q^{2}\left(b^{6} q^{2}-2\right)-6\right)+2 b^{4} q^{2} r^{2}\left(5 b^{6} q^{2}-4\right)-6 b^{2} r^{6}\left(5 b^{6} q^{2}+2\right)\right)\right), \\
\theta_{n}^{3}= & 0, \\
\theta_{n}^{4}= & \frac{b^{7} q^{2} r^{3}}{16\left(b^{6} q^{2}-2\right)^{3}\left(b^{6} q^{2}+1\right)^{2}\left(b^{2} r^{2}-1\right)^{2}\left(-b^{4} q^{2}+b^{2} r^{4}+r^{2}\right)^{2}}( \\
& 18 b^{8} q^{2} r^{4}\left(b^{6} q^{2}-2\right)\left(b^{6} q^{2}+1\right)\left(b^{6} q^{2}+2\right)^{2}+12 b r^{3}\left(b^{6} q^{2}+1\right)^{2}\left(b^{18} q^{6}+14 b^{12} q^{4}+12 b^{6} q^{2}+8\right)+ \\
& +16 b^{4} r^{6}\left(b^{6} q^{2}+1\right)^{2}\left(b^{12} q^{4}+8 b^{6} q^{2}+4\right)+32 b^{4} q^{2}\left(b^{6} q^{2}+1\right)^{2}\left(2 b^{12} q^{4}+b^{6} q^{2}+2\right)+ \\
& -r^{2}\left(b^{6} q^{2}\left(57 b^{24} q^{8}+262 b^{18} q^{6}+436 b^{12} q^{4}+216 b^{6} q^{2}-32\right)+64\right)+
\end{aligned}
$$




$$
\begin{aligned}
& -12 b^{5} r^{7}\left(b^{6} q^{2}+1\right)\left(b^{18} q^{6}+14 b^{12} q^{4}+12 b^{6} q^{2}+8\right)+ \\
& \left.-12 b^{5} q^{2} r\left(b^{6} q^{2}+1\right)\left(3 b^{18} q^{6}+10 b^{12} q^{4}+4 b^{6} q^{2}+24\right)\right)+ \\
& -\frac{b^{2} r^{4} F_{0}}{2\left(b^{6} q^{2}-2\right)\left(b^{6} q^{2}+1\right)\left(b^{6} q^{2} r^{2}-b^{4}\left(q^{2}+r^{6}\right)+r^{2}\right)^{2}}\left(-2 b^{4} q^{2}\left(2+9 b^{6} q^{2}+7 b^{12} q^{4}\right)+\right. \\
& \left.+3 b^{5} q^{2}\left(-4+b^{12} q^{4}\right) r-2 b^{4}\left(2+3 b^{6} q^{2}+b^{12} q^{4}\right) r^{6}+2\left(2+5 b^{6} q^{2}\right)\left(r+b^{6} q^{2} r\right)^{2}\right)+ \\
& -\frac{b^{3} r^{5}\left(2 b^{6} q^{2} r^{2}-3 b^{4} q^{2}+2 r^{2}\right) F_{0}^{2}}{\left(b^{6} q^{2} r^{2}-b^{4}\left(q^{2}+r^{6}\right)+r^{2}\right)^{2}}+\frac{2 b^{5} q r^{4}\left(b^{6} q^{2}+1\right)}{\left(b^{6} q^{2}-2\right)\left(b^{2} r^{2}-1\right)\left(b^{4} q^{2}-b^{2} r^{4}-r^{2}\right)} \frac{\partial F_{0}}{\partial\left(q b^{3}\right)}-\frac{3}{2} L_{4}^{\prime}, \\
\theta_{n}^{5}= & -\frac{3}{2} L_{5}{ }^{\prime}+\frac{F_{0}}{2 b^{2} B r^{2}}+\frac{b^{3} q^{2}\left(-3 b^{7} q^{2} r+4 b^{6} q^{2}-6 b r+4\right)}{8 B r^{3}\left(b^{6} q^{2}-2\right)\left(b^{6} q^{2}+1\right)}, \\
\theta_{n}^{6}= & \frac{\sqrt{3} b^{5} \kappa q^{3}}{2 r^{2}\left(b^{6} q^{2}-2\right)\left(b^{6} q^{2}+1\right)\left(-b^{4} q^{2}+b^{2} r^{4}+r^{2}\right)^{2}}\left(6 b^{15} q^{4} r^{5}+3 b^{13} q^{4} r^{3}+4 b^{12} q^{4} r^{2}+8 b^{10} q^{4}+\right. \\
& \left.+12 b^{9} q^{2} r^{5}-12 b^{8} q^{2} r^{4}+6 b^{7} q^{2} r^{3}+b^{6}\left(12 r^{8}-8 q^{2} r^{2}\right)-4 b^{4}\left(q^{2}-6 r^{6}\right)+24 b^{2} r^{4}+12 r^{2}\right)+ \\
& +\frac{\sqrt{3} b^{6} \kappa q^{3}}{2 r^{2}\left(b^{6} q^{2}+1\right)^{2}\left(b^{2} r^{2}-1\right)\left(-b^{4} q^{2}+b^{2} r^{4}+r^{2}\right)^{2}}( \\
& 12 r\left(-b^{10} q^{4}+b^{8} q^{2} r^{4}+b^{6} q^{2} r^{2}-b^{4} q^{2}+b^{2} r^{4}+r^{2}\right) F_{0}+ \\
& \left.+b^{3} q^{2}\left(b^{4} r^{4}+b^{2} r^{2}+1\right)\left(3 b^{7} q^{2} r-4 b^{6} q^{2}+6 b r-4\right)\right)-\frac{3}{2} L_{6}{ }^{\prime} .
\end{aligned}
$$

\section{E The entropy current at first order}

The authors of reference [3] considered the most general form of entropy current and constitutive relations in hydrodynamics with a charge current (allowing for the possibility of a $U(1)$ anomaly). They showed that at first order in gradients the entropy current must be of the form

$$
S^{\mu}=s u^{\mu}-\frac{\mu}{T} \nu^{\mu}+\frac{1}{2} D l^{\mu},
$$

where $s$ is the equilibrium entropy density, $D$ is a coefficient discussed below, and $\nu^{\mu}$ is the first order correction to the charge current:

$$
J^{\mu}=n u^{\mu}+\nu^{\mu} .
$$

By symmetry arguments this correction can be expressed as

$$
\nu^{\mu}=-\mu \sigma P^{\mu \nu} \partial_{\nu}\left(\frac{\mu}{T}\right)+\frac{1}{2} \xi l^{\mu},
$$

where $\sigma$ and $\xi$ are transport coefficients. For the fluid considered in this paper these transport coefficients can be read off from (C.2). To do this one needs the relations

$$
T=\frac{2-b^{6} q^{2}}{2 \pi b}, \quad \mu=\frac{\sqrt{3} b^{2} q}{\pi},
$$


from which one derives

$$
P^{\mu \nu} \partial_{\nu}\left(\frac{\mu}{T}\right)=-\frac{4 \sqrt{3} b^{3} q\left(b^{12} q^{4}+3 b^{6} q^{2}+2\right)}{\left(b^{6} q^{2}-2\right)^{3}} V_{0}{ }^{\mu} .
$$

The coefficient $\xi$ is non-vanishing only if the theory is anomalous (in the sense that coupling it to a background gauge fields results in an anomalous divergence of the charge current). As shown by Son and Surówka [3], $\xi$ is proportional to the anomaly coefficient $C$ :

$$
\xi=C\left(\mu^{2}-\frac{2}{3} \frac{n \mu^{3}}{\epsilon+p}\right) .
$$

This equation allows one to express $C$ in terms of known quantities, and then one can compute $D$ using the relation [3]

$$
D=\frac{1}{3} C \frac{\mu^{3}}{T} .
$$

Proceeding in this fashion one can express the entropy current (E.1) in terms of the variables $b$ and $q$ used in this paper. This leads to

$$
S^{\mu}=\frac{1}{4 G_{N}}\left(\frac{1}{b^{3}} u^{\mu}-\frac{3 b^{4} q^{2}\left(b^{6} q^{2}+2\right)}{4\left(b^{6} q^{2}-2\right)\left(b^{6} q^{2}+1\right)} V_{0}^{\mu}-\frac{\sqrt{3} b^{7} \kappa q^{3}}{\left(b^{6} q^{2}+1\right)} l^{\mu}\right) .
$$

This is precisely the first order part of (4.1) with coefficients (4.4), which were derived from the holographic entropy current formula (4.2) proposed in [1].

Open Access. This article is distributed under the terms of the Creative Commons Attribution License which permits any use, distribution and reproduction in any medium, provided the original author(s) and source are credited.

\section{References}

[1] I. Booth, M.P. Heller and M. Spalinski, Black Brane Entropy and Hydrodynamics, Phys. Rev. D 83 (2011) 061901 [arXiv: 1010.6301] [InSPIRE].

[2] I. Booth, M.P. Heller, G. Plewa and M. Spalinski, On the apparent horizon in fluid-gravity duality, Phys. Rev. D 83 (2011) 106005 [arXiv:1102.2885] [INSPIRE].

[3] D.T. Son and P. Surowka, Hydrodynamics with Triangle Anomalies, Phys. Rev. Lett. 103 (2009) 191601 [arXiv:0906.5044] [INSPIRE].

[4] J.D. Bekenstein, Black holes and entropy, Phys. Rev. D 7 (1973) 2333 [InSPIRE].

[5] I. Booth and S. Fairhurst, The First law for slowly evolving horizons, Phys. Rev. Lett. 92 (2004) 011102 [gr-qc/0307087] [INSPIRE].

[6] I. Booth, Black hole boundaries, Can. J. Phys. 83 (2005) 1073 [gr-qc/0508107] [InSPIRE].

[7] P. Figueras, V.E. Hubeny, M. Rangamani and S.F. Ross, Dynamical black holes and expanding plasmas, JHEP 04 (2009) 137 [arXiv:0902.4696] [INSPIRE].

[8] I. Booth, M.P. Heller and M. Spalinski, Black brane entropy and hydrodynamics: The Boost-invariant case, Phys. Rev. D 80 (2009) 126013 [arXiv:0910.0748] [INSPIRE]. 
[9] S. Bhattacharyya, V.E. Hubeny, S. Minwalla and M. Rangamani, Nonlinear Fluid Dynamics from Gravity, JHEP 02 (2008) 045 [arXiv:0712.2456] [INSPIRE].

[10] S. Bhattacharyya, V.E. Hubeny, R. Loganayagam, G. Mandal, S. Minwalla et al., Local Fluid Dynamical Entropy from Gravity, JHEP 06 (2008) 055 [arXiv:0803.2526] [INSPIRE].

[11] R. Loganayagam, Entropy Current in Conformal Hydrodynamics, JHEP 05 (2008) 087 [arXiv:0801.3701] [INSPIRE].

[12] L.D. Landau and E.M. Lifshitz, Fluid Mechanics, Second Edition: Volume 6 (Course of Theoretical Physics), Butterworth-Heinemann, 2 ed., January, 1987

[13] P. Romatschke, New Developments in Relativistic Viscous Hydrodynamics, Int. J. Mod. Phys. E 19 (2010) 1 [arXiv:0902.3663] [InSPIRE].

[14] S. Bhattacharyya, Constraints on the second order transport coefficients of an uncharged fluid, JHEP 07 (2012) 104 [arXiv: 1201.4654] [INSPIRE].

[15] G. Compere, P. McFadden, K. Skenderis and M. Taylor, The relativistic fluid dual to vacuum Einstein gravity, JHEP 03 (2012) 076 [arXiv: 1201.2678] [INSPIRE].

[16] J. Erdmenger, M. Haack, M. Kaminski and A. Yarom, Fluid dynamics of R-charged black holes, JHEP 01 (2009) 055 [arXiv:0809.2488] [INSPIRE].

[17] N. Banerjee et al., Hydrodynamics from charged black branes, JHEP 01 (2011) 094 [arXiv:0809.2596] [INSPIRE].

[18] J. Hur, K.K. Kim and S.-J. Sin, Hydrodynamics with conserved current from the gravity dual, JHEP 03 (2009) 036 [arXiv:0809.4541] [INSPIRE].

[19] T. Kalaydzhyan and I. Kirsch, Holographic dual of a boost-invariant plasma with chemical potential, JHEP 02 (2011) 053 [arXiv: 1012.1966] [INSPIRE].

[20] G. Plewa and M. Spalinski, On the gravity dual of strongly coupled charged plasma, JHEP 05 (2013) 002 [arXiv: 1212.2344] [INSPIRE].

[21] A. Chamblin, R. Emparan, C.V. Johnson and R.C. Myers, Charged AdS black holes and catastrophic holography, Phys. Rev. D 60 (1999) 064018 [hep-th/9902170] [INSPIRE].

[22] J.P. Gauntlett, E. O Colgain and O. Varela, Properties of some conformal field theories with M-theory duals, JHEP 02 (2007) 049 [hep-th/0611219] [INSPIRE].

[23] J.P. Gauntlett and O. Varela, Consistent Kaluza-Klein reductions for general supersymmetric AdS solutions, Phys. Rev. D 76 (2007) 126007 [arXiv:0707.2315] [INSPIRE].

[24] S. Bhattacharyya, R. Loganayagam, I. Mandal, S. Minwalla and A. Sharma, Conformal Nonlinear Fluid Dynamics from Gravity in Arbitrary Dimensions, JHEP 12 (2008) 116 [arXiv:0809.4272] [INSPIRE].

[25] S. de Haro, S.N. Solodukhin and K. Skenderis, Holographic reconstruction of space-time and renormalization in the AdS/CFT correspondence, Commun. Math. Phys. 217 (2001) 595 [hep-th/0002230] [INSPIRE].

[26] I. Booth and S. Fairhurst, Isolated, slowly evolving and dynamical trapping horizons: Geometry and mechanics from surface deformations, Phys. Rev. D 75 (2007) 084019 [gr-qc/0610032] [inSPIRE].

[27] P. Romatschke, Relativistic Viscous Fluid Dynamics and Non-Equilibrium Entropy, Class. Quant. Grav. 27 (2010) 025006 [arXiv:0906.4787] [INSPIRE]. 
[28] A. Sadofyev and M. Isachenkov, The Chiral magnetic effect in hydrodynamical approach, Phys. Lett. B 697 (2011) 404 [arXiv: 1010.1550] [InSPIRE].

[29] I. Kanitscheider and K. Skenderis, Universal hydrodynamics of non-conformal branes, JHEP 04 (2009) 062 [arXiv:0901.1487] [INSPIRE].

[30] J. Bhattacharya, S. Bhattacharyya and S. Minwalla, Dissipative Superfluid dynamics from gravity, JHEP 04 (2011) 125 [arXiv: 1101.3332] [INSPIRE].

[31] C.P. Herzog, N. Lisker, P. Surowka and A. Yarom, Transport in holographic superfluids, JHEP 08 (2011) 052 [arXiv:1101.3330] [INSPIRE].

[32] D.E. Kharzeev and H.-U. Yee, Anomalies and time reversal invariance in relativistic hydrodynamics: the second order and higher dimensional formulations, Phys. Rev. D 84 (2011) 045025 [arXiv: 1105.6360] [InSPIRE].

[33] R. Loganayagam, Anomaly Induced Transport in Arbitrary Dimensions, arXiv:1106.0277 [INSPIRE].

[34] K. Peeters, A field-theory motivated approach to symbolic computer algebra, CoRR abs/cs/0608005 (2006).

[35] K. Peeters, Introducing Cadabra: A Symbolic computer algebra system for field theory problems, hep-th/0701238 [INSPIRE].

[36] S. Bhattacharyya et al., Forced Fluid Dynamics from Gravity, JHEP 02 (2009) 018 [arXiv:0806.0006] [INSPIRE]. 